\title{
A role for centromere pairing in meiotic chromosome segregation
}

\author{
Benedict Kemp, ${ }^{1}$ Rebecca Maxfield Boumil, \\ Mara N. Stewart, and Dean S. Dawson ${ }^{2}$
}

Department of Molecular Biology and Microbiology, Tufts

University, Boston, Massachusetts 02111, USA

In meiosis $I$, exchanges provide a connection between homologous chromosome pairs that facilitates their proper attachment to the meiotic spindle. In many eukaryotes, homologous chromosomes that fail to become linked by exchanges exhibit elevated levels of meiotic errors, but they do not segregate randomly, demonstrating that mechanisms beyond exchange can promote proper meiosis I segregation. The experiments described here demonstrate the existence of a meiotic centromere pairing mechanism in budding yeast. This centromere pairing mediates the meiosis I bipolar spindle attachment of nonexchange chromosome pairs and likely plays the same role for all homologous chromosome pairs.

Supplemental material is available at http://www.genesdev.org.

Received May 31, 2004; revised version accepted June 16, 2004.

Exchanges (crossovers), which occur between homologous chromosomes in meiosis I, are critical for the production of viable gametes because they link the homologs together (Bascom-Slack et al. 1997). This linkage allows the homologs to remain joined during the process of microtubule attachment and enables the homologous pair to achieve a bipolar spindle attachment (the centromeres of the homologs attached to microtubules from opposite spindle poles), analogous to sister chromatids in mitotic cells. The importance of recombination in ensuring high-fidelity chromosome segregation might suggest that a pair of nonexchange chromosomes, in an otherwise normal meiosis I, would segregate randomly. However, this is not always the case (Wolf 1994). In several organisms, it has been shown that a single nonexchange pair of chromosomes will be segregated properly in most meioses. Most notably, female Drosophila melanogaster use the pairing of centric heterochromatin to mediate the segregation of the nonexchange fourth chromosome pair (Dernburg et al. 1996; Karpen et al. 1996). In the budding yeast Saccharomyces cerevisiae, a single chromosome pair without an exchange segregates properly in $\sim 90 \%$ of meioses (Dawson et al. 1986; Mann and Davis 1986; Guacci and Kaback 1991; Ross et al. 1996). These examples of nonexchange segregation demonstrate that there are mechanisms, other than exchange,

[Keywords: Meiosis; centromere pairing; nonexchange segregation] ${ }^{1}$ Present address: Department of Biological Sciences, Dartmouth College, Hanover, NH 03755, USA.

${ }^{2}$ Corresponding author.

E-MAIL dean.dawson@tufts.edu; FAX (617) 636-0337.

Article published online ahead of print. Article and publication date are at http://www.genesdev.org/cgi/doi/10.1101/gad.1227304. that contribute to the ability of a chromosome pair to assume a bipolar spindle attachment in meiosis I. The nature of these mechanisms remains largely mysterious. In yeast, nonexchange segregation is not based on DNA sequence homology; numerous studies have shown that nonexchange chromosome pairs that are nonhomologous, even at their centromeres, segregate with the same fidelity $(90 \%)$ as nonexchange pairs that are perfectly homologous (Ross et al. 1996).

The experiments described here explore the mechanisms, beyond exchange, that contribute to meiotic segregation fidelity in $S$. cerevisiae. For these experiments we have constructed a novel yeast strain that has one obligate nonexchange chromosome pair. This strain was created by replacing one copy of $S$. cerevisiae Chromosome $V$ with Chromosome $V$ from Saccharomyces carlsbergensis, which provides full function in haploid $S$. cerevisiae. These "homeologous" chromosomes are $\sim 30 \%$ divergent at the DNA level and virtually never experience meiotic recombination, yet like other yeast nonexchange meiotic chromosome pairs, they segregate from each other in $\sim 90 \%$ of meioses (Boumil et al. 2003).

\section{Results and Discussion}

Synaptonemal complex does not mediate nonexchange segregation

During meiotic prophase, homologous chromosomes are typically linked by both exchanges and synaptonemal complexes (SC). In most eukaryotes, the SC disassembles at diplotene, leaving the chromosomes joined by chiasmata (the cytological manifestation of exchanges). In yeast, diplotene is brief (Padmore et al. 1991), allowing for the possibility that an association of the nonexchange chromosomes, mediated by SC, might persist through diplotene, and orient the centromeres toward opposite poles of the meiotic spindle. This model would be consistent with the demonstration that SC can form between nonhomologous chromosomes (Loidl et al. 1991) and the observation of unusual SC-like structures in cells carrying nonexchange partners (Loidl et al. 1994). We tested this notion by deleting ZIP1, the gene that encodes the central element of the SC (Sym et al. 1993), then scoring the meiotic segregation of the homeologous chromosomes by tetrad analysis. The nondisjunction (both chromosomes moving to the same pole at meiosis I) frequency of the homeologous pair in the zip1 mutant was not distinguishably different from that exhibited by the wild-type control $(5 \%$ vs. $7 \%$ nondisjunction; $n=68$ ). The segregation of the nonexchange chromosomes was not randomized by the absence of SC. Therefore, SC is not critical for dictating nonexchange segregation in budding yeast.

\section{Nonexchange chromosomes experience meiotic centromere pairing}

Models to explain the segregation of nonexchange chromosomes can be divided into those that invoke pairing of the chromosomes versus those that do not (such as counting models in which the cell directs equal numbers of chromosomes to each pole at anaphase). To distinguish between these possibilities, we localized GFP to the homeologs (Straight et al. 1996) and used 
fluorescence microscopy to monitor their interactions in meiotic prophase. The meiotic behavior of the homeologous chromosomes was compared with the interactions of GFP-tagged homologous Chromosome $V \mathrm{~s}$, and to GFP-tagged heterologous chromosomes ( $V$ and $I I I)$.

Meiotic cells were harvested at a time corresponding to pachytene, the stage of maximal homolog pairing, and pairing of the GFP-tagged loci was assayed on chromosome spreads using indirect immunofluorescence. To monitor pairing of the homeologous chromosome arms, GFP was localized to the $I L V 1$ locus, on the right arm of Chromosome $V, 180 \mathrm{~kb}$ from the centromere (Fig. 1a). Tagged loci were scored as "paired" if the centers of the dots were within $0.7 \mu \mathrm{m}$ (Fig. 1b) and unpaired if the dots were farther apart (Fig. 1c).
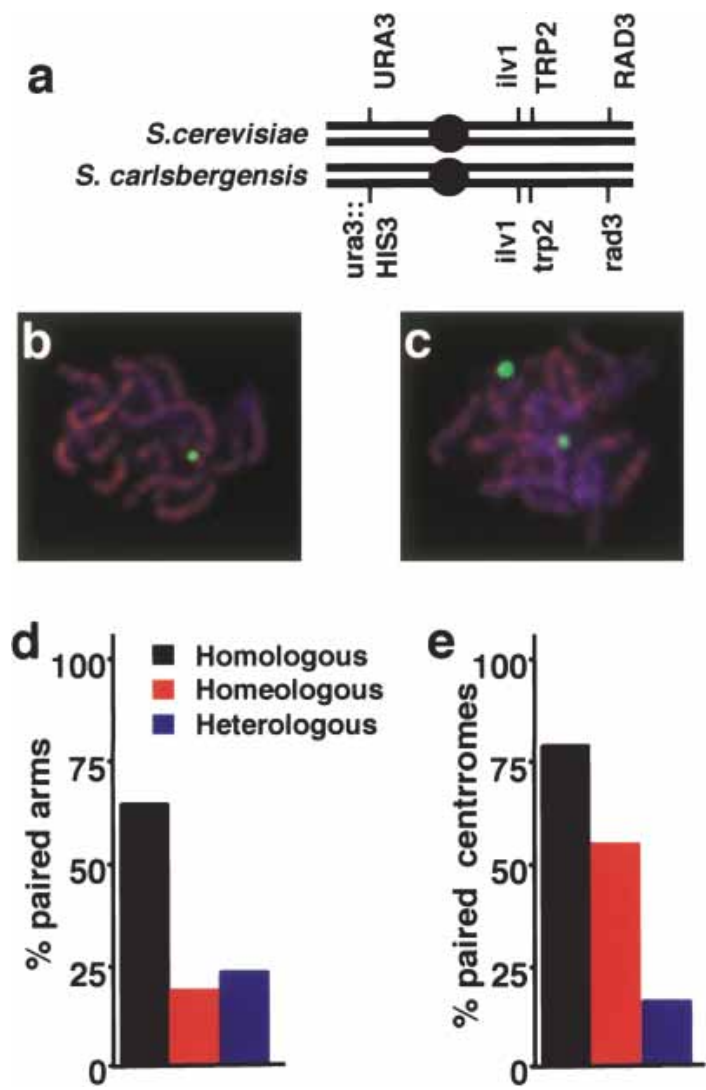

Figure 1. Analysis of pairing of meiotic nonexchange chromosomes in yeast. (a) Maps of the homeologous Chromosome $V$ pair. $(b, c)$ Chromosome spreads from synchronous meiotic culture. (b) An example of a spread with paired GFP dots. (c) An example of unpaired dots. (d) Analysis of chromosome arm pairing. Three diploids were evaluated. Homologous arm pairing was evaluated with a strain bearing a homologous $S$. cerevisiae Chromosome $V$ pair tagged at the ILV1 locus (black bars; DBK215). Heterologous pairing was evaluated with a strain in which one copy of Chromosome $V$ (ILV1) and one copy of Chromosome III (LEU2) was tagged (blue bars; DBK217). Homeologous arm pairing was evaluated in a strain with homeologous Vs tagged at ILV1 (red bars; DBK216). (e) Analysis of chromosome centromere pairing. Homologous centromere pairing was evaluated with a strain bearing a homologous $S$. cerevisiae Chromosome $V$ pair tagged 12 kb from CEN5 (black bars; DBK205). Heterologous pairing was evaluated with a strain in which one copy of Chromosome $V(12 \mathrm{~kb}$ from CEN5) and one copy of Chromosome $I V(12 \mathrm{~kb}$ from CEN4) was tagged (blue bars; DBK201). Homeologous centromere pairing was evaluated in a strain with both homeologous Vs tagged $12 \mathrm{~kb}$ from CEN5 (red bars; DBK203).
Homologous Chromosome $V$ arms were scored as paired in $>63 \%$ of the meiotic chromosome spreads examined (Fig. 1d, black bars). Homologous arms synapse in virtually $100 \%$ of meioses; thus, this assay underestimates the true level of pairing. The failure to observe $100 \%$ pairing could be due to disruption of pairing by the spreading technique or may be attributable to the fact that synapsis is not synchronous and some of the spreads we examined might not have yet synapsed the Chromosome $V$ pair. In contrast, both the heterologous arms and the homeologous arms were separated in $>80 \%$ of nuclei (Fig. 1d). These data suggest that whereas the arms of homologous chromosomes are aligned in pachytene, the arms of the homeologous chromosomes are no more likely to be paired than the arms of two heterologous chromosomes (each with its own homologous partner). In both the heterologous and homeologous strains we observed a fraction $(-20 \%)$ of nuclei with only one visible dot. It is likely this does not represent true pairing but instead may be caused by juxtaposition of dots by random chance, failure to detect one of the dots for technical reasons, or low levels of association of the GFP-lacI clusters (Aragon-Alcaide and Strunnikov 2000).

Very different results were obtained when GFP was localized to a position $12 \mathrm{~kb}$ from the centromere of Chromosome $V$. Both homologous and homeologous centromeres were paired in pachytene spreads $177 \%$ and $54 \%$, respectively; Fig. 1e). Therefore, unlike the arms, the centromere regions of the homeologous chromosomes are paired in pachytene. In contrast, the centromeres of heterologous chromosomes were nearly always separated (>80\%; Fig. 1e).

To test whether the pachytene centromere pairing of the nonexchange chromosomes is the basis of their disjunction, we took advantage of a previously described aspect of nonexchange segregation. Genetic experiments have shown that the segregation fidelity of a nonexchange pair is disrupted when a third nonexchange chromosome or centromere plasmid is introduced into the strain (Dawson et al. 1986; Guacci and Kaback 1991; Ross et al. 1996). The three nonexchange chromosomes typically act as nearly partners. That is, the segregation behavior of three nonexchange chromosomes is consistent with the model that, in each meiosis, any two of the three chromosomes can be chosen as partners and moved to opposite poles, while the third chromosome segregates randomly. This behavior predicts three kinds of meiosis I segregation patterns that should occur with nearly equal frequency if the chromosomes are all equally likely to pair, and this is what is observed (Fig. 2a). The homeologous Chromosome Vs have a slight preference for each other as partners, probably because of their extensive sequence homology (Boumil et al. 2003), and typically exhibit $\sim 25 \%$ nondisjunction (Fig. 2a, Type C) in the presence of a third nonexchange chromosome or centromere plasmid. If centromere pairing is the basis of the nonexchange segregation, then introduction of a third nonexchange chromosome should lower pachytene centromere pairing of the homeologous chromosomes, presumably by pairing with one of the two homeologs, and consequently raising nondisjunction of the homeologs. This is what we observed when a centromere plasmid (CEN6) was added to a strain bearing the homeologous pair. The plasmid acted as a competitor and increased nondisjunction of the homeologs from $7 \%$ to $25 \%$, consistent with previous studies (Boumil et al. 


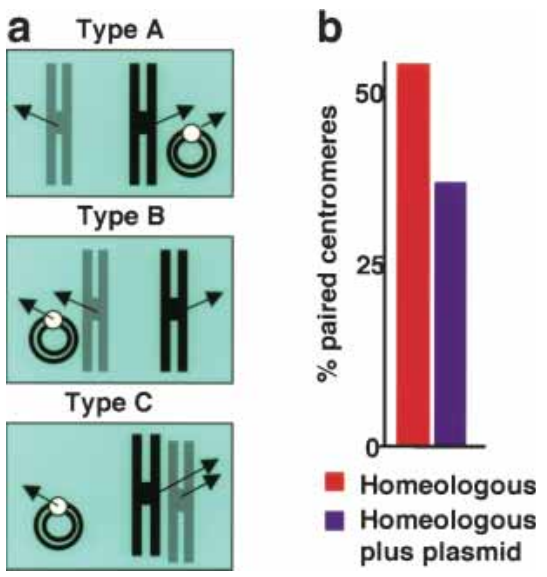

Figure 2. Disruption of centromere pairing with a competing nonhomologous centromere. $(a)$ The three observed meiosis I segregation outcomes in cells with three nonexchange chromosomes; in this case two homeologous Chromosome Vs and a CEN6 plasmid (pRK21). In each outcome, the single chromosome going to one pole has presumably paired with one of the two chromosomes that have gone to the opposite pole, while the third has segregated randomly. (b) Pairing of GFP-tagged homeologous centromeres in strain DBK203 with or without the competitor plasmid. The evaluation of centromere pairing was as described in Figure 1. (Red bar) Without competitor; (purple bar) with competitor.

2003). Cytological analysis of the pairing of the GFPtagged homeologous centromeres in this strain showed that the competing centromere plasmid decreased homeologous centromere pairing $17 \%$ (from $54 \%$ to $37 \%$; Fig. $2 \mathrm{~b}$ ), which mirrors the $18 \%$ increase in nondisjunction caused by the competitor plasmid. This is the result that would be predicted if the centromere plasmid is able to participate as a pairing partner with the homeologous chromosomes and nonexchange segregation is driven by this centromere pairing. An alternate explanation (addressed below) is that the extra centromere plasmid indirectly disrupts meiotic behavior of the homeologous chromosome pair.

\section{Meiotic centromere pairing is DNA-sequence-independent}

We tested whether a centromere plasmid disrupts the segregation of the homeologous chromosomes directly, by pairing with them, by assaying pairing interactions between the homeologous chromosomes and a competing yeast artificial chromosome (YAC) bearing CEN3. The methods used were similar to those used for studying pairing of the homeologous Vs except that the YAC was visualized using a myc-epitope tag, and one of the homeologous $V$ pair (the $S$. cerevisiae $V$ ) was tagged with GFP. The 65-kb YAC (YLp61; Ross et al. 1992) is composed mostly of bacteriophage $\lambda$ DNA and carries a $5-\mathrm{kb}$ interval of Chromosome III that includes CEN3. The YAC was myc-tagged by introducing a tet operator array $3 \mathrm{~kb}$ from the CEN3. We then introduced into these cells a construct that would express a TetR-13xmyc hybrid protein that would localize to the tetracycline operator arrays and allow detection of the YAC with antibodies against the myc-epitope. The $S$. cerevisiae Chromosome $V$ was GFP-tagged at $U R A 3(\sim 35 \mathrm{~kb}$ from
CEN5). Cells bearing the homeologous Chromosome $V$ pair and the YAC were induced to undergo meiosis, chromosome spreads were prepared, and the positions of the YAC and Chromosome $V$ "dots" were determined using indirect immunofluorescence microscopy. The relative positions of the myc (YAC) and GFP (Chromosome V) dots were measured in chromosome spreads of cells that were determined to be in late prophase by DAPI staining (the condensed chromosomes give a wormy appearance; Supplementary Fig. 1). Tetrad analysis revealed that the YAC elevated the nondisjunction of the homeologs to $24 \%$. If all of these nondisjunctions occur when the centromere of one of the homeologous Chromosome Vs pairs with the centromere of the YAC, then this predicts the YAC pairs with each homeolog in $\sim 24 \%$ of meioses (Fig. 3a). In these cases of pairing between the YAC and one of the Chromosome Vs, there is a $50 \%$ chance that the unpaired homeolog will segregate to the same pole as its homeolog, yielding a nondisjunction. When the pairing of the YAC with the GFP-tagged Chromosome $V$ was monitored in meiotic chromosome spreads, we observed that they were paired in $\sim 19 \%$ of the spreads (Fig. 3b). This value is slightly below the predicted $24 \%$, but note that with homologous centromeres that are synapsed in virtually $100 \%$ of meioses (Fig. 1e), we only detect $77 \%$ of the pairing with this method. As a control, the pairing of the YAC with the GFP-tagged Chromosome $V$ when it had a homologous (exchange) partner was assayed using the same methods. In this experiment we observed pairing of the GFP and myc signals in only $6 \%$ of meioses (Fig. 3b), a value significantly less than the $19 \%$ pairing observed when the Chromosome $V$ did not have an exchange partner $(p<0.001)$. These results demonstrate first, that nonhomologous centromeres can be paired in meiosis I, and second, that as in the experiments with homologous and homeologous tagged centromeres, there is a correlation between the level of centromere pairing of the centromere plasmid and Chromosome $V$ and their level of disjunction, the result expected if centromere pairing leads to disjunction.

If centromeres are able to pair in a DNA-sequenceindependent fashion, then what prevents random pairing

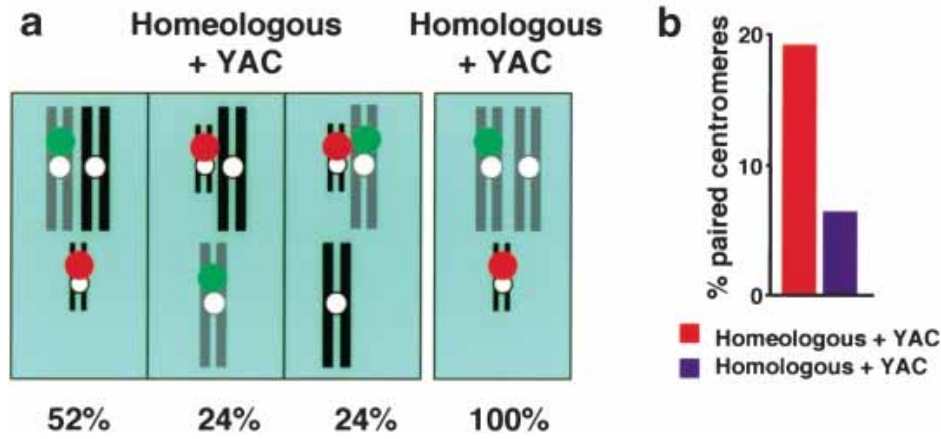

Figure 3. Analysis of pairing of a YAC with an S. cerevisiae Chromosome V. Experiments were performed with isogenic strains carrying a GFP-tagged Chromosome $V$ and a myc-tagged YAC. $(a)$ The predicted meiosis I pairing outcomes in cells with three nonexchange chromosomes, either two homeologous Chromosome Vs and a YAC (left panel), or two homologous Chromosome Vs and the YAC (right panel). (b) The level of pairing that was observed in meiotic chromosome spreads between a myc-tagged YAC (3 kb from CEN3) and a $S$. cerevisiae Chromosome $V$, GFP-tagged at the URA3 locus $35 \mathrm{~kb}$ from CEN5, in the presence of a homeologous Chromosome $V$ (red bar; DMS173), or a homologous Chromosome $V$ (purple bar; DMS174; $n=101$ for each strain). 
of the centromeres? The ability of an exchange partner to block the pairing of the YAC and the GFP-tagged Chromosome $V$ suggests that it is exchange, and the synapsis that follows exchange, that juxtaposes homologous centromeres and blocks random centromere pairing.

\section{Paired centromeres separate precociously at metaphase I}

Chromosome pairs that have achieved a bipolar spindle attachment remain linked by crossovers until the signal to proceed into anaphase, at which time dissolution of sister-chromatid cohesion, distal to the crossover, permits the chromosomes to separate (for review, see Cohen-Fix 2000). To test whether the centromere association of the nonexchange chromosomes is sufficient to maintain the linkage of homeologs until anaphase, we examined the positioning of the homeologous chromosomes on the metaphase spindle. The similar morphologies of metaphase and early anaphase spindles in meiosis I of $S$. cerevisiae make them difficult to distinguish. To circumvent this problem, cells were delayed in metaphase I by expressing a nondegradable form of Pds1p $(P D S 1-\Delta d b)$ under the control of a meiotic promoter $\left(\mathrm{P}_{I M E 2}\right.$; Shonn et al. 2000). In these cells, we monitored positioning of the centromere-associated GFPtags on both the homologous and homeologous Chromosome $V$ pairs.

On the meiosis I metaphase spindles, $91 \%$ of the homologous centromere $V s$ appeared as a single (paired) dot (Fig. 4a). The homologous centromeres are likely under tension and may even be pulled toward the poles as is seen in mitotic cells /Goshima and Yanagida 2000; He et al. 2000), but because the GFP tag is $\sim 12 \mathrm{~kb}$ from the centromere, this stretching might not be detected by our assay. Conversely, only $24 \%$ of the homeologous GFPtagged centromeres were paired. Instead, most $(76 \%)$ had separated to give two dots (Fig. 4b). The positions of the centromeres on the metaphase spindles were measured and plotted as a function of spindle length (Fig. 4c,d). The paired homologous centromeres were usually positioned between the spindle poles (Fig. 4c; each orange circle represents the position of the paired centromeres in one cell). Instances in which the GFP dots were separated were limited to long spindles (Fig. 4c; each separated pair of GFP dots is represented by a pair of light- and darkblue diamonds). In these cases, the separated dots were usually located on opposite ends of the spindle. Because the cells eventually escape the Pds $1-\Delta$ db-induced metaphase delay after several hours (data not shown), the spindles with separated homologous centromeres (Fig. $4 \mathrm{c}$, blue diamonds) may represent those that have proceeded into anaphase I.

In contrast, the homeologous centromeres were separated and positioned very close to the spindle poles in most metaphase cells (Fig. 4d, blue diamonds), demonstrating that the chromosomes had not simply drifted apart, but instead were actively disjoined by spindle-mediated forces. The fact that the nonexchange chromosomes are separated on even the shortest spindles suggests that they are separated, in most cases to opposite poles, immediately upon attachment to microtubules. In $6 \%$ (6 of 100) of the cells, both homeologous centromeres were at one spindle pole (Fig. 4d, pairs of black squares; see upper right spindle in Fig. $4 \mathrm{~b}$ as an example). This was never seen for the homologous centromeres and likely corresponds to the $7 \%$ meiosis I nondisjunction exhibited by these nonexchange chromosomes.

\section{Centromere pairing and nonexchange segregation}

The above experiments suggest the basis of nonexchange segregation in $S$. cerevisiae. We propose that centromere regions in yeast are able to undergo meiotic pairing that is sequence-independent, and that this pairing orients the kinetochores of the nonexchange partners such that they are likely to encounter microtubules that radiate from opposite spindle poles. The fact that the pairing is able to orient the kinetochores, coupled with the fact that even core centromere regions of $125 \mathrm{bp}$ support nonexchange segregation, suggests that the centromere pairing occurs at, or very close to, the actual kinetochores. These results suggest a model for nonexchange segregation (Fig. 5). Cells enter meiosis with decondensed chromatin and their centromeres clustered (Hayashi et al. 1998). In prophase, condensation begins and homologous recombination triggers the synapsis of homologous partners, which pulls their centromeres away from the centromere cluster (Fig. 5, center; Hayashi et al. 

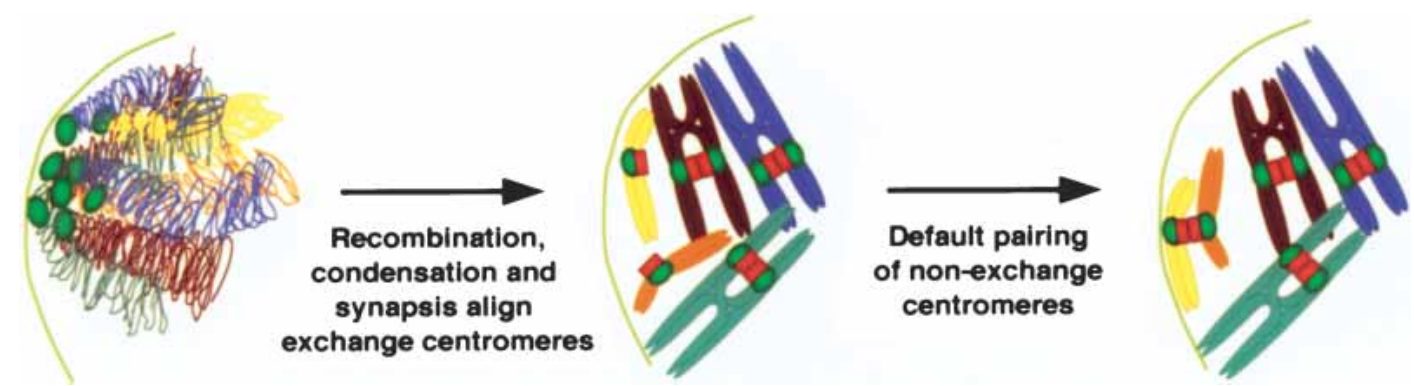

Figure 5. Model for centromere pairing in meiosis I. Cells enter meiosis I with decondensed chromosomes and clustered centromeres (green ovals; Hayashi et al. 1998). Recombination between homologs, followed by condensation and synapsis, pulls homologous centromeres into juxtaposition, mediating their pairing. Nonexchange centromeres pair by default, as all other potential pairing partners have been removed by synapsis. In wild-type cells, it is unlikely that more than one chromosome pair will fail to recombine. Thus, in these cells the default centromere pairing will mediate the segregation of homologous chromosomes even though the process is sequence-independent. As a consequence of centromere pairing, the microtubule attachment sites for each bivalent face in opposite directions.

1998; R. Shanks and D.S. Dawson, unpubl.), leaving the centromeres of the nonexchange chromosomes (Fig. 5, yellow and orange chromosomes) as default partners, rather like attractive students at a school dance pairing off, leaving the two least popular students as partners.

The yeast nonexchange segregation mechanism bears both similarity to and differences from the nonexchange process described in Drosophila females (Dernburg et al. 1996; Karpen et al. 1996). In Drosophila, pairing of centric heterochromatin appears to mediate the segregation of homologous nonexchange chromosomes. Unlike the yeast system, this pairing is homology-dependent. It is yet unclear how the pairing of nonexchange chromosomes ensures their disjunction in Drosophila; however, the pairing is not of kinetochores, per se, but of adjacent chromosomal regions. It has been proposed that the pairing may play a role similar to chiasmata, by providing a link that opposes the pull of microtubules (Dernburg et al. 1996).

\section{The meiotic role of centromere pairing}

A mechanism for partitioning nonexchange chromosomes would not be needed in most budding yeast meioses, as nonexchange chromosomes are probably rare (Kaback et al. 1992). Why, then, does the mechanism exist? Although homologous centromeres are clearly juxtaposed when homologs experience end-to-end meiotic alignment in pachytene, there has been little evidence in yeast to suggest that the centromeres undergo active pairing, rather than simply being positioned next to one another by the process of synapsis. We suggest that the centromere pairing seen here with the homeologous pair also occurs between the centromere regions of homologous chromosomes (Fig. 5, red rectangles).

What might be the role of meiotic centromere pairing? Early studies of meiotic chromosome dynamics led Östergren (1951) to suggest that pairing of the centromeres of homologous chromosomes might outwardly orient the kinetochores, optimizing the chances for bipolar spindle attachment (as shown in Fig. 5). Our results suggest that, in yeast, the proper segregation of nonexchange chromosomes occurs by this type of process. This mechanism would increase the probability of an initial bipolar spindle attachment of all chromosome pairs, not just nonexchange chromosomes. Genetic evidence for such a model has been provided by the obser- vation that the segregation fidelity of exchange chromosome fragments is improved if their centromeres are in register upon homologous alignment (Guerra and Kaback 1999). Chromosome pairs that have experienced exchanges can experience tension at their kinetochores when they attach to microtubules from opposite poles. Pairs with inappropriate attachments fail to experience this tension, which leads to a checkpoint-mediated signal that leads to a delay (Li and Nicklas 1995), during which chromosomes can reorient on the spindle (Nicklas and Koch 1969). In budding yeast, exchange chromosomes can benefit from this process (Shonn et al. 2000), but nonexchange chromosomes probably cannot. Thus, meiotic centromere pairing can be thought of as an alternative to the tension-mediated mechanism for attaining a bipolar spindle attachment. This mechanism is clearly critical for the segregation of nonexchange chromosomes, but may contribute to the segregation of exchange chromosomes as well.

In humans, such a mechanism could have special significance, as many birth defects are the result of errant segregation of nonexchange chromosomes (Hassold and Hunt 2001). In humans, as in yeast, these nonexchange chromosomes presumably cannot benefit from the tension-based mechanisms that ensure proper spindle attachments for exchange chromosomes. Instead, the segregation of these error-prone chromosomes must either be left to chance, or dependent on alternative processes like the centromere pairing described here in yeast.

\section{Materials and methods}

Genetic manipulation of yeast

Alteration of the genome by transformation with DNA was performed using published methods (Burke et al. 2000). Tetrad analysis was used to monitor the effect of a competitor plasmid on segregation of the homeologous chromosome pair using previously described methods (Boumil et al. 2003).

Tetrad analysis was performed using published methods (Burke et al. 2000). All strains were derived from S288C progenitors. The genotypes are in Supplementary Table 1.

Meiotic chromosome pairing assays

Clusters of the lac operon operator sequence or the tet operon operator sequence were targeted to specific chromosomal loci using established methods (Straight et al. 1996; Michaelis et al. 1997). Diploids contained

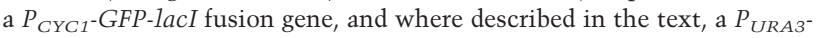
tetR-13xmyc gene for detecting lac operator and tet operator arrays, respectively. Meiotic chromosome spreads were prepared using previously 
described methods (Kamieniecki et al. 2000). Chromosome pairing was evaluated in spreads with multiple worm-like structures visualized with either, antibodies directed against the Zip1 synaptonemal complex protein, or by DAPI staining. GFP-tagged or myc-tagged chromosomes were visualized using indirect fluorescence microscopy. GFP was detected with either rabbit or chicken anti-GFP (Chemicon) antibody, and the myc-epitope was detected using mouse 9E10 monoclonal antibody. Secondary antibodies were FITC-conjugated donkey anti-rabbit (Jackson), Alexa 488 goat anti-chicken, and Alexa goat anti-mouse (Molecular Probes) antibodies. Dots with centers that were within $0.7 \mu \mathrm{m}$ were scored as paired. Images were collected and processed using a Hamamatsu CCD and OpenLabs 3.0 software.

Analysis of centromere position in metaphase

A PDS1 open reading frame with a deletion of the coding region for the destruction box (Shonn et al. 2000) was placed under the control of the meiotic IME2 promoter. This construct was integrated into the genome, leaving both native copies of PDS1 intact. Cells bearing the $P_{I M E 2^{-}} P D S 1$ $\Delta d b$ construct showed no vegetative growth defects. Cells were harvested from synchronous meiotic cultures $24 \mathrm{~h}$ after induction of meiosis. Cells were fixed with formaldehyde, and indirect immunofluorescence was used to visualize tubulin and GFP using previously described methods (Kamieniecki et al. 2000).

\section{Acknowledgments}

We are grateful to Michael Lichten, Rebecca Kamieniecki, and Peter Cheslock for comments on the manuscript; Michael Lichten, Dan Burke, Orna Cohen-Fix, David Kaback, Robert Shivers, Lauren Logsden, and members of the Dawson laboratory for helpful discussions of the research; Chip Quinn for the high school dance analogy; and Heather Coffin for technical assistance. Aaron Straight and Andrew Murray generously provided reagents and advice for GFP-tagging chromosomes. Marion Shonn provided the PDS1- $\Delta \mathrm{db}$ plasmid, and Susannah Rankin provided anti-myc antibodies. The research was supported by NSF grant MCB-0078138.

The publication costs of this article were defrayed in part by payment of page charges. This article must therefore be hereby marked "advertisement" in accordance with 18 USC section 1734 solely to indicate this fact.

\section{References}

Aragon-Alcaide, L. and Strunnikov, A.V. 2000. Functional dissection of in vivo interchromosome association in Saccharomyces cerevisiae. Nat. Cell Biol. 2: 812-818.

Bascom-Slack, C.A., Ross, L.O., and Dawson, D.S. 1997. Chiasmata, crossovers, and meiotic chromosome segregation. In Advances in genetics (eds. J. Hall and J. Dunlap), pp. 253-284. Academic Press, Inc., San Diego.

Boumil, R.M., Kemp, B., Angelichio, M., Nilsson-Tillgren, T., and Dawson, D.S. 2003. Meiotic segregation of a homologous chromosome pair. Mol. Genet. Genomics 268: 750-760.

Burke, D., Dawson, D., and Stearns, T. 2000. Methods in yeast genetics: A Cold Spring Harbor laboratory course manual. Cold Spring Harbor Laboratory Press, Cold Spring Harbor, NY.

Cohen-Fix, O. 2000. Sister chromatid separation: Falling apart at the seams. Curr. Biol. 10: R816-R819.

Dawson, D., Murray, A.W., and Szostak, J.W. 1986. An alternate pathway for meiotic chromosome segregation in yeast. Science 234: 713-717.

Dernburg, A.F., Sedat, J.W., and Hawley, R.S. 1996. Direct evidence of a role for heterochromatin in meiotic chromosome segregation. Cell 86: $135-146$.

Goshima, G. and Yanagida, M. 2000. Establishing biorientation occurs with precocious separation of the sister kinetochores, but not the arms, in the early spindle of budding yeast. Cell 100: 619-633.

Guacci, V. and Kaback, D.B. 1991. Distributive disjunction of authentic chromosomes in Saccharomyces cerevisiae. Genetics 127: 475-488.

Guerra, C.E. and Kaback, D.B. 1999. The role of centromere alignment in meiosis I segregation of homologous chromosomes in Saccharomyces cerevisiae. Genetics 153: 1547-1560.

Hassold, T. and Hunt, P. 2001. To err (meiotically) is human: The genesis of human aneuploidy. Nat. Rev. Genet. 2: 280-291.

Hayashi, A., Ogawa, H., Kohno, K., Gasser, S.M., and Hiraoka, Y. 1998. Meiotic behaviours of chromosomes and microtubules in budding yeast: Relocalization of centromeres and telomeres during meiotic prophase. Genes Cells 3: 587-601.

He, X., Asthana, S., and Sorger, P.K. 2000. Transient sister chromatid separation and elastic deformation of chromosomes during mitosis in budding yeast. Cell 101: 763-775.

Kaback, D.B., Guacci, V., Barber, D., and Mahon, J.W. 1992. Chromosome size-dependent control of meiotic recombination. Science 256: $228-232$.

Kamieniecki, R.J., Shanks, R.M.Q., and Dawson, D.S. 2000. Slk19p is necessary to prevent separation of sister chromatids in meiosis I. Curr. Biol. 10: 1182-1190.

Karpen, G.H., Le, M.H., and Le, H. 1996. Centric heterochromatin and the efficiency of achiasmate disjunction in Drosophila female meiosis. Science 273: 118-122.

Li, X. and Nicklas, B. 1995. Mitotic forces control cell-cycle checkpoint. Nature 373: 630-632.

Loidl, J., Nairz, K., and Klein, F. 1991. Meiotic chromosome synapsis in a haploid yeast. Chromosoma 100: 221-228.

Loidl, J., Scherthan, H., and Kaback, D.B. 1994. Physical association between nonhomologous chromosomes precedes distributive disjunction in yeast. Proc. Natl. Acad. Sci. 91: 331-334.

Mann, C. and Davis, R.W. 1986. Meiotic disjunction of circular minichromosomes in yeast does not require DNA homology. Proc. Natl. Acad. Sci. 83: 6017-6019.

Michaelis, C., Ciosk, R., and Nasmyth, K. 1997. Cohesins: Chromosomal proteins that prevent premature separation of sister chromatids. Cell 91: $35-45$.

Nicklas, R.B. and Koch, C.A. 1969. Chromosome manipulation III Spindle fiber tension and the re-orientation of mal-oriented chromosomes. J. Cell Biol. 43: 40-50.

Östergren, G. 1951. The mechanism of co-orientation in bivalents and multivalents. Heriditas 37: 85-156.

Padmore, R., Cao, L., and Kleckner, N. 1991. Temporal comparison of recombination and synaptonemal complex formation during meiosis in S. cerevisiae. Cell 66: 1239-1256.

Ross, L.O., Treco, D., Nicolas, A., Szostak, J.W., and Dawson, D. 1992 Meiotic recombination on artificial chromosomes in yeast. Genetics 131: $541-550$.

Ross, L.O., Rankin, S., Flatters, M., and Dawson, D. 1996. Effects of homology, size and exchange on the meiotic segregation of model chromosomes in Saccharomyces cerevisiae. Genetics 142: 79-89.

Shonn, M.A., McCarroll, R., and Murray, A.W. 2000. Requirement of the spindle checkpoint for proper chromosome segregation in budding yeast meiosis. Science 289: 300-303.

Straight, A.F., Belmont, A.S., Robinett, C.C., and Murray, A.W. 1996. GFP tagging of budding yeast chromosomes reveals that protein-protein interactions can mediate sister chromatid cohesion. Curr. Biol. 6: 1599-1608.

Sym, M., Engebrecht, J., and Roeder, G.S. 1993. ZIP1 is a synaptonemal complex protein required for meiotic chromosome synapsis. Cell 72: 365-378.

Wolf, K.W. 1994. How meiotic cells deal with non-exchange chromosomes. Bioessays 16: 107-114. 


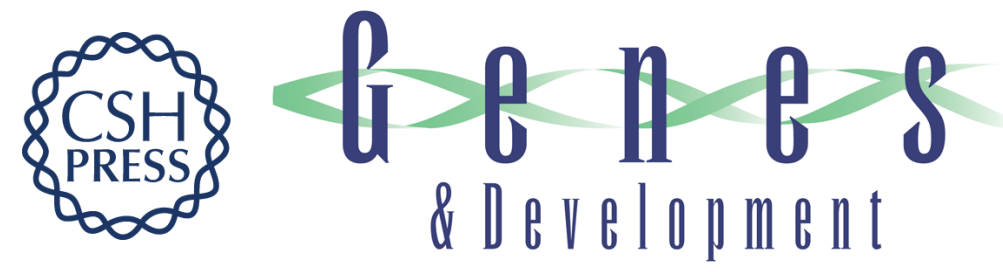

\section{A role for centromere pairing in meiotic chromosome segregation}

Benedict Kemp, Rebecca Maxfield Boumil, Mara N. Stewart, et al.

Genes Dev. 2004, 18:

Access the most recent version at doi:10.1101/gad.1227304

Supplemental

Material

References

This article cites 28 articles, 11 of which can be accessed free at: http://genesdev.cshlp.org/content/18/16/1946.full.html\#ref-list-1

\section{License}

Email Alerting Service

http://genesdev.cshlp.org/content/suppl/2004/08/03/1227304.DC1 right corner of the article or click here.

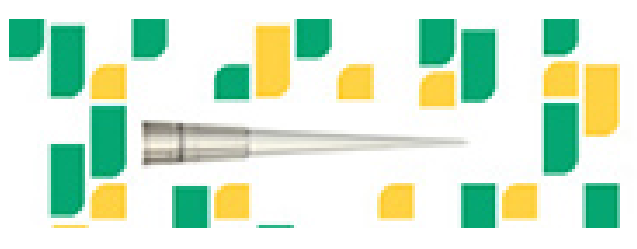

Focused on your science. 